\title{
Illicit and Prescription Drug Misuse among Sexual Minority Women in the United States: A Protocol for a Scoping Review
}

\author{
Kelly W. Gagnon ${ }^{1 *}$, Jessica Frankeberger ${ }^{1 *}$, Stephanie Corey ${ }^{2}$, Barbara L. Folb ${ }^{1,3}$, Christina \\ Mair $^{1}$, Helena VonVille ${ }^{1,3}$, Robert W. S. Coulter ${ }^{1,4}$ \\ ${ }^{1}$ Department of Behavioral and Community Health Sciences, Graduate School of Public Health, \\ University of Pittsburgh, Pittsburgh, USA \\ ${ }^{2}$ Department of Epidemiology, Graduate School of Public Health, \\ University of Pittsburgh, Pittsburgh, USA \\ ${ }^{3}$ Health Sciences Library System, University of Pittsburgh, Pittsburgh, USA \\ ${ }^{4}$ Department of Pediatrics, School of Medicine, University of Pittsburgh, Pittsburgh, USA
}

\begin{abstract}
Background: The United States Institute of Medicine (IOM) published reports in 1999 and 2011 identifying drug use as a priority area for sexual minority health research, specifically focused on mechanisms contributing to drug use disparities and the development of tailored interventions. Limited research has prioritized sexual minority women (SMW) with the majority of substance use research among sexual minorities focused on adolescents and men who have sex with men. This scoping review will characterize the research literature related to illicit drug use and prescription drug misuse among SMW and sub-groups within the SMW population. Through this, we will identify: (1) specific substances used; (2) patterns of substance use; (3) risk and protective factors; (4) prevention interventions; and (5) drug treatment programs specific to SMW.
\end{abstract}

Methods/Design: This review will include studies with empirical data of illicit or prescription drug misuse among sexual minority women. Peer-reviewed quantitative research conducted in the United States and published in English from 2011-Present will be included. We will search Medline, PsycINFO, and Web of Science databases for relevant articles. Two independent reviewers will screen abstracts and relevant full-text studies for eligibility. Data will be extracted from eligible articles and results will be presented in narrative and tabular form as appropriate.

Discussion: This work will identify gaps of knowledge in the research pertaining to illicit or prescription drug misuse among sexual minority women since the 2011 US IOM report. As a result of this work, we will propose directions for future research to address identified gaps.

Keywords: drug use; substance use, lesbian and bisexual, sexual minority women, women who have sex with women

\footnotetext{
* Correspondence to Kelly W. Gagnon, ${ }^{1}$ Department of Behavioral and Community Health Sciences, Graduate School of Public Health, University of Pittsburgh, Pittsburgh, USA. Email: keg118@pitt.edu

Social Science Protocols, December 2020, 1-12.

http://dx.doi.org/10.7565/ssp.v3.5050
} 


\section{Background}

Substance use continues to be a prominent public health concern in the United States (US) (Centers for Disease Control and Prevention, 2019; Substance Abuse and Mental Health Services Administration, 2018). Research shows that marginalized and stigmatized populations, such as sexual minorities, are disproportionately at higher risk for using licit and illicit substances (Johns et al., 2018; Mennis \& Stahler, 2016). Sexual minorities comprise individuals who identify their sexual orientation as non-heterosexual (e.g., lesbian, gay, bisexual, or queer), report same-sex sexual behavior, or same-sex sexual attraction. Female, male, and transgender sexual minorities experience unique stressors and contexts based on their gender and sexuality that impact their physical and mental health (Schuler et al., 2019). These stressors are often the result of stigma and discrimination related to being a sexual minority (Goldbach et al., 2014). Minority stress can be the source of social and emotional distress resulting in substance use as a coping mechanism (Goldbach et al., 2014; Lehavot \& Simoni, 2011). The adverse outcomes of minority stress are augmented by other socioecological factors, including higher rates of unemployment and lower levels of health insurance coverage among sexual minorities (Charlton et al., 2018).

To date, sexual minority men have been the predominant focus of sexual minority research, particularly in the area of substance use (Coulter et al., 2014; Heath et al., 2012; Melendez-Torres \& Bourne, 2016; Vosburgh et al., 2012). With a higher proportion of women $(5.1 \%)$ identifying as a sexual minority compared to men $(3.9 \%)$ and more women $(12.6 \%)$ reporting sexual acts with same-sex partners than men $(2.8 \%)$, the dearth of research and understanding of substance use among sexual minority women (SMW) is concerning (Copen et al., 2016; Newport, 2018).

In 1999, the US Institute of Medicine (IOM) Committee on Lesbian, Gay, Bisexual, and Transgender Health Issues published a report on the health of lesbians in the US, finding that substance use was a pressing health issue for SMW and identifying major gaps in existing knowledge and research (Solarz, 1999). While this report noted substantial limitations in the research literature, preliminary evidence suggested that lesbian women reported higher use of cocaine, inhalants, and marijuana compared to heterosexual women (Solarz, 1999). Ultimately, the 1999 report called for more extensive research on drug use patterns and risks among SMW to further understand the patterns and context of problematic use within this often-overlooked population.

In 2011, the IOM published an updated report on the health of sexual and gender minorities and still identified substance use as a particularly critical and understudied issue (Graham et al., 2011). The 2011 report found that there were distinct differences in subgroups of sexual minorities' substance use and abuse (Graham et al., 2011). More specifically, adolescent bisexual girls, when compared to both heterosexual and other sexual minority boys and girls, were the most likely to have tried marijuana and other illicit drugs (Graham et al., 2011). Similar findings were reported among adults, with women who have sex with women more likely to report injection drug use compared to women who only have sex with men (Graham et al., 2011). Overall, substance use disparities by sexual minority status were also greater for SMW compared to sexual minority men (Graham et al., 2011). Although the findings of this thorough review of existing research suggested heightened sexuality differences in use for women, the report had only a limited discussion of other risk factors or determinants of drug use disparities in SMW.

The objective of this scoping review is to build upon previous efforts to understand the current state of research on drug use behaviors among SMW by exploring research on illicit drug use conducted in the US since the 2011 IOM report. Specifically, we will assess the 
current knowledge regarding drug use for SMW. We will identify gaps in the existing literature and propose new directions for research and public health efforts moving forward.

We conducted a preliminary search of databases [PubMed (National Library of Medicine), APA PsycInfo, Prospero, and Joanna Briggs Institute Database of Systematic Reviews and Implementation Reports] to ensure this work is not duplicative of previously published reviews or protocols. Despite the two IOM reports highlighting drug use disparities among SMW, we discovered that a limited understanding of the risk and protective factors, and existing prevention and intervention efforts for drug use among SMW still remains. Research analyzing substance use among sexual minorities has traditionally focused on disparities in alcohol and tobacco use with less emphasis on illicit drugs and prescription drug misuse (Hughes et al., 2020; Talley et al., 2016). This was reflected in a recent scoping review of substance use among SMW globally (Hughes et al., 2020). The principle findings of this scoping review focused on alcohol use with limited mention of specific other drug use (Hughes et al., 2020). As a result, we still have a limited understanding of the specific illicit drug use and prescription drug misuse patterns, social determinants, or interventions among SMW in the US (Meyer et al., 2019).

We believe that a scoping review of drug use among SMW is warranted, especially given the duration of time since the last major review of the literature. This scoping review will characterize the research literature related to drug use and prescription drug misuse among SMW and sub-groups within the SMW population. Through this, we will identify: (1) specific substances used; (2) patterns of substance use; (3) risk and protective factors; (4) prevention interventions; and (5) drug treatment programs specific to SMW. Only those studies conducted with SMW living in the United States and territories and published since 2011 will be included in this scoping review.

\section{Methods/Design}

The JBI Manual for Evidence Synthesis (Peters et al., 2017) was used to guide the development of this scoping review. Study execution was assessed using the appropriate criteria from the AMSTAR 2.0 checklist (Faggion, 2015). The PRISMA Extension for Scoping Reviews (PRISMA-ScR) (Tricco et al., 2018) was followed to write this review.

\subsection{Inclusion criteria}

This scoping review aims to understand the progress of the field of drug use research among SMW. The inclusion criteria presented in Table 1 are reflective of the key attributes of studies related to our primary objective to determine the state of research almost 10 years after the 2011 IOM report. Each of the inclusion criteria are described in detail below.

Table 1. Inclusion criteria.

\begin{tabular}{ll}
\hline Category & Criteria \\
\hline Types of participants & Cisgender sexual minority females \\
Outcomes & Illicit drug use or prescription drug misuse \\
Context & United States and US territories \\
Types of Sources & Quantitative, empirical peer-reviewed journal articles published in \\
& English since 2011 \\
\hline
\end{tabular}




\subsubsection{Types of participants}

This review will consider studies that explicitly identify SMW participants in the United States. For the purposes of this review, SMW is defined as cisgender women who either identify as a sexual minority (i.e., lesbian, bisexual, queer, etc.) and/or report same-sex sexual behavior and/or attraction. We will be inclusive with regard to the inclusion of SMW, including sexual orientation, behavior, and attraction as indicators of sexual minority status because individuals from disadvantaged and/or racial/ethnic minority communities that engage in same-sex behavior are less likely to identify as a sexual minority and individuals who engage in same-sex sexual behavior, regardless of sexual identity, often experience similar discrimination and stressors as those who identify as gay, lesbian, bisexual, or queer (Copen et al., 2016; Lindley et al., 2012; McCabe et al., 2005; Teasdale \& Bradley-Engen, 2010). All age and race/ethnicity groups will be included in this review.

This scoping review will exclude articles that exclusively focus on gender minorities (i.e., transgender or nonbinary individuals). Because gender minorities have unique experiences from cisgender women that may influence their substance use behaviors, these factors are beyond the scope of this review (James et al., 2016). A separate review should be conducted that takes into consideration these experiences and the unique social and political contexts surrounding gender minorities and substance use.

\subsubsection{Outcomes}

This review will include studies that examine illicit drug use or prescription drug misuse as the outcome variable. As such, studies examining drug use patterns, correlates, risk and protective factors, and interventions and treatment programs for drug use specifically among SMW will be included. Studies will be included if they provide empirical quantitative data regarding drug use outcomes in this population.

For the purposes of this review, illicit drug use includes the consumption by any method (e.g., injecting, inhaling, smoking) of illegal substances (e.g., cocaine, heroin, inhalants, methamphetamines, hallucinogens), regardless of dosage or frequency. As marijuana/cannabis remains a prohibited substance in many U.S. states as well as at the federal level as of the time of this review, it will be included as an illicit substance. However, cannabis used appropriately for medicinal purposes will be excluded. Prescription drug misuse will include the consumption of any prescription drug (e.g., opioids, benzodiazepines, stimulants, medicinal cannabis) that is used either without a prescription from a healthcare provider or misused from the way it was originally prescribed. Studies exclusively focused on alcohol or tobacco use will be excluded. Measures of general substance use which do not identify specific drugs will be included unless alcohol and/or tobacco are included in the measure.

\subsubsection{Context}

This review will consider studies that include results from individuals in the United States or the US territories (American Samoa, Guam, the Northern Mariana Islands, Puerto Rico and the U.S. Virgin Islands). This review is limited to the United States due to specific and significant historical and policy issues surrounding both sexual minority populations and illicit substance use (Flores, 2014; Killian, 2010; MacCoun \& Reuter, 2011; Werb et al., 2011). 


\subsubsection{Types of sources}

This scoping review will consider articles in the peer-reviewed scientific literature. Studies presenting quantitative empirical and original data will be included. Articles presenting only qualitative data, literature review articles, research or intervention protocols, commentaries, opinion articles, or other types of publications that do not present empirical, quantitative results will be excluded. Clinical and individual case studies will also be excluded.

This review will be limited to articles published in English from 2011 to the present. As described previously, the year 2011 was selected due to the publication of IOM report on sexual and gender minority health, which presented a thorough examination of substance use among SMW through 2010 (Graham et al., 2011). As such, this review aims to build on the IOM's previous work and examine the state of drug use research among SMW in the time following the release of this report.

\subsection{Search strategy}

The databases to be searched include Medline (PubMed), PsycINFO, and Web of Science. The search will be limited to peer-reviewed journals and articles published from 2011Present. After conducting a database search for relevant literature and identifying over 1800 peer-reviewed studies, it was determined that gray literature will be excluded considering the number of studies included in the analysis.

The search strategy will aim to locate published, peer-reviewed articles in electronic databases. An initial limited search strategy was developed and pilot tested by a health research librarian on Medline (PubMed) to identify articles on the topic. The text words contained in the titles and abstracts of relevant articles, the index terms used to describe the articles, and medical subject headings (e.g., MeSH terms) were used to develop a full search strategy (see Appendix I). The basic search strategy includes the concepts SMW and illicit and prescription drugs. Filters for English language, human subjects, and the United States were applied. The full search strategy, including all identified keywords and index terms, will be adapted for each included information source (Medline, PyschINFO, and Web of Science).

\subsection{Study selection}

Following the search, all identified records will be collated and uploaded into DistillerSR (Evidence Partners, Ottawa, Canada) and duplicates will be removed. Titles and abstracts will then be screened independently by two reviewers for assessment against the inclusion criteria. Potentially relevant papers will be retrieved in full and their citation details imported into DistillerSR. The full text of selected citations will then be assessed in detail against the inclusion criteria by two independent reviewers to confirm eligibility. Reasons for exclusion of full-text articles will be documented and reported in the scoping review. Any discrepancies that arise between the reviewers at each stage of the selection process will be resolved through discussion or by consulting a third reviewer. The results of the search and study selection process will be reported in full in the final scoping review and presented in a flow diagram as described by the Preferred Reporting Items for Systematic Reviews and Metaanalyses Extension for Scoping Reviews (PRISMA-ScR) (Tricco et al., 2018).

\subsection{Data extraction}

Data will be extracted from papers included in the scoping review using a data extraction tool developed by the reviewers. A draft extraction tool is provided in Appendix II. The draft data extraction tool will be modified and revised as necessary during this process. 
Modifications will be detailed in the full scoping review. Any disagreements that arise between the reviewers will be resolved through discussion or by consulting a third reviewer.

Two primary categories of studies will be created: 1) observational studies (research examining drug use patterns, risk/protective factors, and/or health and social factors associated with drug use), and 2) intervention or program evaluation studies in which drug use is the outcome. Within each of these two categories, the data extracted, as appropriate, will include specific details about the sample size and characteristics (e.g., age, race/ethnicity), study focus and objectives, types of drugs examined and measurement of use, measurement of SMW, and key findings.

\section{Presentation of results}

This scoping review aims to present an overview of existing research on illicit and prescription drug use among SMW in the United States since the 2011 IOM report (Graham et al., 2011). The extracted data will be presented in a narrative form, accompanied by tables and figures as appropriate, in a manner that aligns with this scoping review's objective. Data will be presented in two tables, one for each category of studies (observational studies and intervention studies). Within these tables, findings will be stratified by drug examined. Additionally, PRISMA-ScR will be used to guide the presentation of the results to ensure systematic and comprehensive reporting (Tricco et al., 2018).

\section{Declarations}

Funding: This research was funded in part by the National Institute on Alcohol Abuse and Alcoholism (K01AA027564 to RWSC through the University of Pittsburgh) and National Center For Advancing Translational Sciences of the National Institutes of Health (TL1TR001858 to RWSC and KG). The content is solely the responsibility of the authors and does not necessarily represent the official views of the NIH. The NIH was not involved in this article's study design, analysis or interpretation of data, the writing of the report, or the decision to submit the article for publication.

Competing interests: The authors declare that they have no competing interests.

\section{References}

Centers for Disease Control and Prevention. (2019). Illicit Drug Use. https://www.cdc.gov/nchs/fastats/drug-use-illicit.htm

Charlton, B. M., Gordon, A. R., Reisner, S. L., Sarda, V., Samnaliev, M., \& Austin, S. B. (2018). Sexual orientation-related disparities in employment, health insurance, healthcare access and health-related quality of life: a cohort study of US male and female adolescents and young adults. BMJ Open, 8(6). https://doi.org/10.1136/bmjopen-2017-020418

Copen, C. E., Chandra, A., \& Febo-Vazquez, I. (2016). Sexual behavior, sexual attraction, and sexual orientation among adults aged 18-44 in the United States: data from the 20112003 National Survey of Family Growth. National Health Statistics Reports, (88), 1-14.

Coulter, R. W., Kenst, K. S., \& Bowen, D. J. (2014). Research funded by the National Institutes of Health on the health of lesbian, gay, bisexual, and transgender populations. American Journal of Public Health, 104(2). https://doi.org/10.2105/AJPH.2013.301501 
Faggion, C. M. (2015). Critical appraisal of AMSTAR: challenges, limitations, and potential solutions from the perspective of an assessor. BMC Medical Research Methodology, 15(1), 1-5. https://doi.org/10.1186/s12874-015-0062-6

Flores, A. (2014). National Trends in Public Opinion on LGBT Rights in the United States. The Williams Institute. https://escholarship.org/uc/item/72t8q7pg

Goldbach, J. T., Tanner-Smith, E. E., Bagwell, M., \& Dunlap, S. (2014). Minority stress and substance use in sexual minority adolescents: A meta-analysis. Prevention Science, 15(3), 350-363. https://doi.org/10.1007/s11121-013-0393-7

Graham, R., Berkowitz, B., Blum, R., Bockting, W., Bradford, J., de Vries, B., \& Makadon, H. (2011). The health of lesbian, gay, bisexual, and transgender people: Building a foundation for better understanding. Institute of Medicine, 10, 13128.

Heath, J., Lanoye, A., \& Maisto, S. A. (2012). The role of alcohol and substance use in risky sexual behavior among older men who have sex with men: a review and critique of the current literature. AIDS and Behavior, 16(3), 578-589. https://doi.org/10.1007/s10461011-9921-2

Hughes, T. L., Veldhuis, C. B., Drabble, L. A., \& Wilsnack, S. C. (2020). Research on alcohol and other drug (AOD) use among sexual minority women: A global scoping review. PloS One, 15(3). https://doi.org/10.1371/journal.pone.0229869

Johns, M. M., Lowry, R., Rasberry, C. N., Dunville, R., Robin, L., Pampati, S., Stone, D. M., \& Kollar, L. M. M. (2018). Violence victimization, substance use, and suicide risk among sexual minority high school students - United States, 2015-2017. Morbidity and Mortality Weekly Report, 67(43), 1211. https://doi.org/ 10.15585/mmwr.mm6743a4

Killian, M. L. (2010). The political is personal: Relationship recognition policies in the United States and their impact on services for LGBT people. Journal of Gay \& Lesbian Social Services, 22(1-2), 9-21. https://doi.org/10.1080/10538720903332149

Lehavot, K., \& Simoni, J. M. (2011). The impact of minority stress on mental health and substance use among sexual minority women. Journal of Consulting and Clinical Psychology, 79(2), 159. https://doi.org/ 10.1037/a0022839

Lindley, L. L., Walsemann, K. M., \& Carter Jr, J. W. (2012). The association of sexual orientation measures with young adults' health-related outcomes. American Journal of Public Health, 102(6), 1177-1185. https://doi.org/ 10.2105/AJPH.2011.300262

MacCoun, R. J., \& Reuter, P. (2011). Assessing drug prohibition and its alternatives: A guide for agnostics. Annual Review of Law and Social Science, 7, 61-78. https://doi.org/10.1146/annurev-lawsocsci-102510-105442

McCabe, S. E., Hughes, T. L., Bostwick, W., \& Boyd, C. J. (2005). Assessment of difference in dimensions of sexual orientation: implications for substance use research in a collegeage population. Journal of Studies on Alcohol, 66(5), 620-629. https://doi.org/10.15288/jsa.2005.66.620

Melendez-Torres, G., \& Bourne, A. (2016). Illicit drug use and its association with sexual risk behaviour among MSM: more questions than answers? Current Opinion in Infectious Diseases, 29(1), 58-63. https://doi.org/ 10.1097/QCO.0000000000000234

Mennis, J., \& Stahler, G. J. (2016). Racial and ethnic disparities in outpatient substance use disorder treatment episode completion for different substances. Journal of Substance Abuse Treatment, 63, 25-33. https://doi.org/10.1016/j.jsat.2015.12.007

Meyer, J. P., Isaacs, K., El-Shahawy, O., Burlew, A. K., \& Wechsberg, W. (2019). Research on women with substance use disorders: Reviewing progress and developing a research and implementation roadmap. Drug and Alcohol Dependence.

https://doi.org/10.1016/j.drugalcdep.2019.01.017

Newport, F. (2018). In U.S., Estimate of LGBT Population Rises to 4.5\%. Gallup. https://news.gallup.com/poll/234863/estimate-lgbt-population-rises.aspx

Social Science Protocols, December 2020, 1-12.

http://dx.doi.org/10.7565/ssp.v3.5050 
Peters, M., Godfrey, C., \& McInerney, P. (2017). Chapter 11: Scoping Reviews, Joanna Briggs Institute Reviewer Manual. The Joanna Briggs Institute.

Schuler, M. S., Stein, B. D., \& Collins, R. L. (2019). Differences in substance use disparities across age groups in a national cross-sectional survey of lesbian, gay, and bisexual adults. LGBT Health, 6(2), 68-76. https://doi.org/ 10.1089/lgbt.2018.0125

Solarz, A. L. (1999). Lesbian health: Current Assessment and Directions for the Future. National Academies Press.

Substance Abuse and Mental Health Services Administration. (2018). Substance Use Data Provide Roadmap for Future Action. https://www.samhsa.gov/data/release/2017-nationalsurvey-drug-use-and-health-nsduh-releases

Talley, A. E., Gilbert, P. A., Mitchell, J., Goldbach, J., Marshall, B. D., \& Kaysen, D. (2016). Addressing gaps on risk and resilience factors for alcohol use outcomes in sexual and gender minority populations. Drug and Alcohol Review, 35(4), 484-493. https://doi.org/ 10.1111/dar. 12387

Teasdale, B., \& Bradley-Engen, M. S. (2010). Adolescent same-sex attraction and mental health: The role of stress and support. Journal of Homosexuality, 57(2), 287-309. https://doi.org/10.1080/00918360903489127

Tricco, A. C., Lillie, E., Zarin, W., O'Brien, K. K., Colquhoun, H., Levac, D., Moher, D., Peters, M. D., Horsley, T., \& Weeks, L. (2018). PRISMA extension for scoping reviews (PRISMA-ScR): checklist and explanation. Annals of Internal Medicine, 169(7), 467-473. https://doi.org/10.7326/M18-0850

Vosburgh, H. W., Mansergh, G., Sullivan, P. S., \& Purcell, D. W. (2012). A review of the literature on event-level substance use and sexual risk behavior among men who have sex with men. AIDS and Behavior, 16(6), 1394-1410. https://doi.org/10.1007/s10461-0110131-8

Werb, D., Rowell, G., Guyatt, G., Kerr, T., Montaner, J., \& Wood, E. (2011). Effect of drug law enforcement on drug market violence: A systematic review. International Journal of Drug Policy, 22(2), 87-94. https://doi.org/:10.1016/j.drugpo.2011.02.002 
Appendix I. Search strategy for PubMed.

Search conducted on March 5, 2019

\begin{tabular}{|c|c|c|}
\hline Number & Search Terms & Items found \\
\hline \#14 & Limit \#13 to 2011-Present & 994 \\
\hline \#13 & Search (\#11 AND \#12) & 1854 \\
\hline \#12 & $\begin{array}{l}\text { Search ((Alabama[TIAB] OR Alaska[TIAB] OR Arizona[TIAB] OR } \\
\text { Arkansas[TIAB] OR California[TIAB] OR Colorado[TIAB] OR } \\
\text { Connecticut[TIAB] OR Delaware[TIAB] OR District of } \\
\text { Columbia[TIAB] OR "Washington, DC"[TIAB] OR Florida[TIAB] OR } \\
\text { Georgia[TIAB] OR Hawaii[TIAB] OR Idaho[TIAB] OR Illinois[TIAB] } \\
\text { OR Indiana[TIAB] OR Iowa[TIAB] OR Kansas[TIAB] OR } \\
\text { Kentucky[TIAB] OR Louisiana[TIAB] OR Maine[TIAB] OR } \\
\text { Maryland[TIAB] OR Massachusetts[TIAB] OR Michigan[TIAB] OR } \\
\text { Minnesota[TIAB] OR Mississippi[TIAB] OR Missouri[TIAB] OR } \\
\text { Montana[TIAB] OR Nebraska[TIAB] OR Nevada[TIAB] OR "New } \\
\text { Hampshire"[TIAB] OR "New Jersey"[TIAB] OR "New Mexico"[TIAB] } \\
\text { OR "New York"[TIAB] OR "North Carolina"[TIAB] OR "North } \\
\text { Dakota"[TIAB] OR Ohio[TIAB] OR Oklahoma[TIAB] OR } \\
\text { Oregon[TIAB] OR Pennsylvania[TIAB] OR "Rhode Island"[TIAB] OR } \\
\text { "South Carolina"[TIAB] OR "South Dakota"[TIAB] OR } \\
\text { Tennessee[TIAB] OR Texas[TIAB] OR Utah[TIAB] OR } \\
\text { Vermont[TIAB] OR Virginia[TIAB] OR Washington[TIAB] OR "West } \\
\text { Virginia"[TIAB] OR Wisconsin[TIAB] OR Wyoming[TIAB] OR } \\
\text { United States[TIAB] OR U.S.[tiab] OR USA[tiab] OR U.S.A.[tiab])) } \\
\text { OR (("United States"[MeSH] OR United States[PL] OR United } \\
\text { States[GR])) OR ((United States[AD] OR USA[AD] OR U.S.A.[AD] } \\
\text { OR U.S.[AD] OR Alabama[AD] OR Alaska[AD] OR Arizona[AD] OR } \\
\text { Arkansas[AD] OR California[AD] OR Colorado[AD] OR } \\
\text { Connecticut[AD] OR Delaware[AD] OR District of Columbia[AD] OR } \\
\text { "Washington, DC"[AD] OR Florida[AD] OR Georgia[AD] OR } \\
\text { Hawaii[AD] OR Idaho[AD] OR Illinois[AD] OR Indiana[AD] OR } \\
\text { Iowa[AD] OR Kansas[AD] OR Kentucky[AD] OR Louisiana[AD] OR } \\
\text { Maine[AD] OR Maryland[AD] OR Massachusetts[AD] OR } \\
\text { Michigan[AD] OR Minnesota[AD] OR Mississippi[AD] OR } \\
\text { Missouri[AD] OR Montana[AD] OR Nebraska[AD] OR Nevada[AD] } \\
\text { OR "New Hampshire"[AD] OR "New Jersey"[AD] OR "New } \\
\text { Mexico"[AD] OR "New York"[AD] OR "North Carolina"[AD] OR } \\
\text { "North Dakota"[AD] OR Ohio[AD] OR Oklahoma[AD] OR } \\
\text { Oregon[AD] OR Pennsylvania[AD] OR "Rhode Island"[AD] OR "South } \\
\text { Carolina"[AD] OR "South Dakota"[AD] OR Tennessee[AD] OR } \\
\text { Texas[AD] OR Utah[AD] OR Vermont[AD] OR Virginia[AD] OR } \\
\text { Washington[AD] OR "West Virginia"[AD] OR Wisconsin[AD] OR } \\
\text { Wyoming[AD] AND (English[lang]))) }\end{array}$ & $14,323,987$ \\
\hline$\# 11$ & Search (\#9 NOT \#10) & 2,723 \\
\hline
\end{tabular}




\begin{tabular}{|c|c|c|}
\hline$\# 10$ & Search (animals[mh] NOT (animals[mh] AND humans[mh])) & $4,553,519$ \\
\hline$\# 9$ & Search (\#7 AND \#8) & 2,728 \\
\hline \#8 & Search English[Language] & $24,781,459$ \\
\hline \#7 & Search (\#5 AND \#6) & 3,091 \\
\hline \#6 & $\begin{array}{l}\text { sh:noexp] OR Substance Withdrawal Syndrome[mh] OR addict*[tiab] } \\
\text { OR amphetamine*[tiab] OR cannabis[tiab] OR Chetiab] OR } \\
\text { cocaine[tiab] OR drug abuse*[tiab] OR drug misuse* OR drug } \\
\text { use*[tiab] OR heroin[tiab] OR illicit drug*[tiab] OR marijuana[tiab] OR } \\
\text { narcotic*[tiab] OR opiate*[tiab] OR opioid*[tiab] OR recreational } \\
\text { drug*[tiab] OR Street drug*[tiab]) }\end{array}$ & 381,739 \\
\hline$\# 5$ & Search (\#1 OR \#4) & 19,469 \\
\hline$\# 4$ & Search (\#2 AND \#3) & 17,341 \\
\hline$\# 3$ & $\begin{array}{l}\text { Search (Female[Mesh] OR Female*[tiab] OR Woman*[tiab] OR } \\
\text { Women*[tiab]) }\end{array}$ & $8,497,304$ \\
\hline \#2 & $\begin{array}{l}\text { Search ((Bisexuality[MeSH Terms] OR Homosexuality[mh:noexp] OR } \\
\text { Sexual and Gender Minorities[mh:noexp] OR bicurious[tiab] OR } \\
\text { bisexual[tiab] OR bisexuality[tiab] OR bisexuals[tiab] OR gay[tiab] OR } \\
\text { gays[tiab] OR Gender minorit*[tiab] OR gender queer[tiab] OR } \\
\text { genderqueer[tiab] OR GLB[tiab] OR GLBQ[tiab] OR GLBs[tiab] OR } \\
\text { GLBT[tiab] OR GLBTQ[tiab] OR heteroflexible[tiab] OR homo } \\
\text { sex*[tiab] OR homosexual[tiab] OR homosexualities[tiab] OR } \\
\text { homosexuality[tiab] OR homosexuals[tiab] OR LGB[tiab] OR } \\
\text { LGBQ[tiab] OR LGBS[tiab] OR LGBT[tiab] OR queer[tiab] OR same } \\
\text { gender loving[tiab] OR same sex attracted[tiab] OR Same sex } \\
\text { behavior[tiab] OR same sex couple*[tiab] OR Same sex experience[tiab] } \\
\text { OR same sex relations[tiab] OR Same sex sexualit*[tiab] OR sexual } \\
\text { identity[tiab] OR sexual minorities[tiab] OR sexual minority[tiab] OR } \\
\text { sexual orientation[tiab] OR sexual preference[tiab]) NOT (laparoscopic } \\
\text { gastric bypass[tiab] OR gay[au])) }\end{array}$ & 34,704 \\
\hline$\# 1$ & $\begin{array}{l}\text { Search (Homosexuality, female[MeSH Terms] OR lesbian[tiab] OR } \\
\text { lesbianism[tiab] OR lesbians[tiab] OR lesbigay[tiab] OR women loving } \\
\text { women[tiab] OR women who have sex with women[tiab] OR } \\
\text { WSW[tiab] OR WSWM[tiab]) }\end{array}$ & 7,011 \\
\hline
\end{tabular}


Appendix II. Data extraction instrument.

\begin{tabular}{|l|l|}
\hline \multicolumn{2}{|l|}{ Article and Study Characteristics: } \\
\hline Author(s) & \\
\hline Year of Publication & \\
\hline Study Primary Purpose/Objective: & \\
\hline Study Design (e.g., Cohort, Cross-sectional): & \\
\hline Theoretical Framework: & \\
\hline Location of Study: & \\
\hline Recruitment Setting (e.g., hospital, drug treatment): & \\
\hline Sampling Design (e.g., random, convenience): & \\
\hline Data Collection Methods: & \\
\hline SMW Sample Characteristics: & \\
\hline Sample Size - Total: & \\
\hline Sample Size - Sexual Minority Women: & \\
\hline Sample Age Range: & \\
\hline $\begin{array}{l}\text { Age Range of Sexual Minority Women (if } \\
\text { presented): }\end{array}$ & \\
\hline Sample Race/Ethnicity Composition: & \\
\hline Race/Ethnicity Composition of Sexual Minority \\
Women (if presented): & \\
\hline Inclusion/Exclusion Criteria: & \\
\hline Measurement/Analysis: & \\
\hline Identification and measurement of sexual minority & \\
\hline Drugs Examined: & \\
\hline
\end{tabular}




\begin{tabular}{|l|l|}
\hline Measurement of Drug Use: & \\
\hline $\begin{array}{l}\text { Primary Outcome of Study and Measurement of } \\
\text { Variable: }\end{array}$ & \\
\hline $\begin{array}{l}\text { Primary Independent Variable and Measurement of } \\
\text { Variable: }\end{array}$ & \\
\hline Covariates Examined: & \\
\hline $\begin{array}{l}\text { Intervention or Drug Treatment Description (if } \\
\text { applicable): }\end{array}$ & \\
\hline Qualifying drug outcome(s) among SMW: & \\
\hline
\end{tabular}

